\title{
A Labile Metallo-Porphyrin as Tool to Control J-Aggregates Chiroptical Properties
}

\author{
Created by: Luigi Monsù Scolaro ${ }^{1}$, llaria Occhiuto ${ }^{2}$, Mariachiara Trapani ${ }^{3}$,
} Roberto Zagami ${ }^{4}$, Andrea Romeo ${ }^{5}$, Maria Angela Castriciano 6

1, Dipartimento di Scienze Chimiche, Biologiche, Farmaceutiche ed Ambientali , Università di Messina, V.le F. Stagno D'Alcontres, 31-98166, Messina, Italy; Imonsu@unime.it

2, Dipartimento di Scienze Chimiche, Biologiche, Farmaceutiche ed Ambientali , Università di Messina, V.le F. Stagno D'Alcontres, 31-98166, Messina, Italy; ilaria.occhiuto@gmail.com

3, CNR-ISMN Istituto per lo Studio dei Materiali Nanostrutturati c/o Dipartimento di Scienze Chimiche, Biologiche, Farmaceutiche ed Ambientali, University of Messina, V.le F. Stagno D'Alcontres, 31-98166 Messina, Italy; mariachiara.trapani@cnr.it

4, CNR-ISMN Istituto per lo Studio dei Materiali Nanostrutturati c/o Dipartimento di Scienze Chimiche, Biologiche, Farmaceutiche ed Ambientali, University of Messina, V.le F. Stagno D'Alcontres, 31-98166 Messina, Italy; roberto.zagami@ismn.cnr.it

5, Dipartimento di Scienze Chimiche, Biologiche, Farmaceutiche ed Ambientali , Università di Messina, V.le F. Stagno D'Alcontres, 31-98166, Messina, Italy;

anromeo@unime.it

6, CNR - ISMN Istituto per lo Studio dei Materiali Nanostrutturati c/o Dipartimento di Scienze Chimiche, Biologiche, Farmaceutiche ed Ambientali, University of Messina, V.le F. Stagno D'Alcontres, 31-98166 Messina, Italy; maria.castriciano@cnr.it

Version received: 15 July 2020

check for updates

The zinc(II) metal derivative of 5,10,15,20-tetrakis(4-sulfonatophenyl)porphyrin (TPPS 4 ) is quite labile and readily demetallates under acidic conditions, affording the parent diacid porphyrin in a monomeric form. The rate of this process is first order on $\left[\mathrm{ZnTPPS}_{4}\right]$ and second order on $\left[\mathrm{H}^{+}\right]$, allowing a precise control of the monomer release in solution. Under high ionic strength, this latter species is able to self-assemble into J-aggregates, whose kinetics of growth are largely modulated by $\mathrm{pH}$. The aggregation kinetics have been treated according to a well-established model, in which the formation of an initial nucleus is the rate determining step preceding the autocatalytic growth of the whole assembly. The extinction spectra of the aggregates suggest the occurrence of a dipolar coupling mechanism very similar to that operating in metal nanoparticles. Spontaneous symmetry breaking takes place in these aggregates as evidenced by unusual circular dichroism spectra. The intensity and sign of the effect is controlled by the aggregation rate and therefore can be tuned through a proper choice of initial conditions.

J-aggregates are supramolecular systems in which the constituent chromophore units are laterally arranged. Apart the occurrence of a specific J-band in the UV/Vis spectra, bathochromically shifted with respect to the free monomer, a series of peculiar opto-electronic and chiroptical properties attracted the interest of many researchers on these species in the last decades []][ㄹ] . Usual candidates to build such structures are aromatic compounds, e.g. porphyrins and, among them the water soluble 5,10,15,20-tetrakis(4-sulfonatophenyl)porphyrin $\left(\mathrm{TPPS}_{4}\right.$ ) has been intensely studied. Such interest arises from the possibility of obtaining a variety of supramolecular architectures whose size spans from the

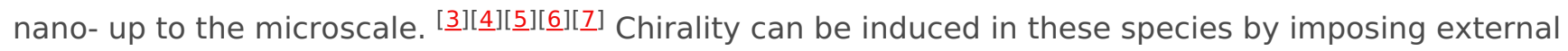

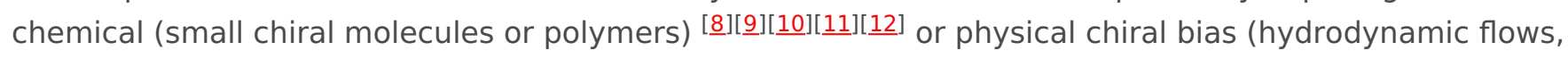

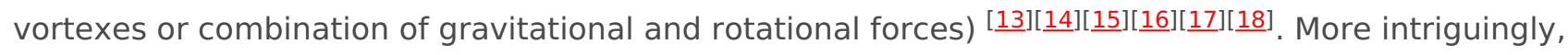
spontaneous symmetry breaking can also occur in the absence of any bias, leading to a search for the

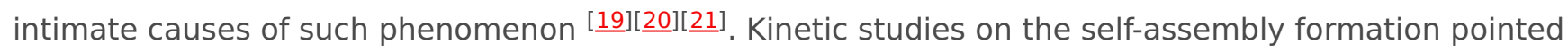
out the role of aggregation rates and the mixing protocol on the intensity of the circular dichroic signals [22]. Furthermore, these findings suggest that the interplay of local concentration and temperature gradients, mixing techniques, and also aging of reagents could have a deep impact on the 
reproducibility of the experiments, since the distribution of species in solution at the beginning of aggregation could be unpredictable (monomers, dimers, oligomers etc.). In complex systems, all these factors are able to change the aggregation pathway and eventually influence the resulting structure of the final supramolecular assembly [23]. For these reasons, it is very important to develop approaches to minimize the potential sources of uncertainty. In a previous study, we showed that controlled amounts of the zinc(II) metal derivative of $\mathrm{TPPS}_{4}$ when added to the metal-free porphyrin decrease consistently the formation of J-aggregates and influence their chiroptical properties [녀]. This particular metal complex is quite labile under acidic conditions and the metal ion can be easily removed, [ㄷ] affording an easy and convenient way to obtain in situ the monomeric diacid $\mathrm{H}_{2}$ TPPS $_{4}$, that is the building block for the subsequent aggregate growth (see Scheme 1).

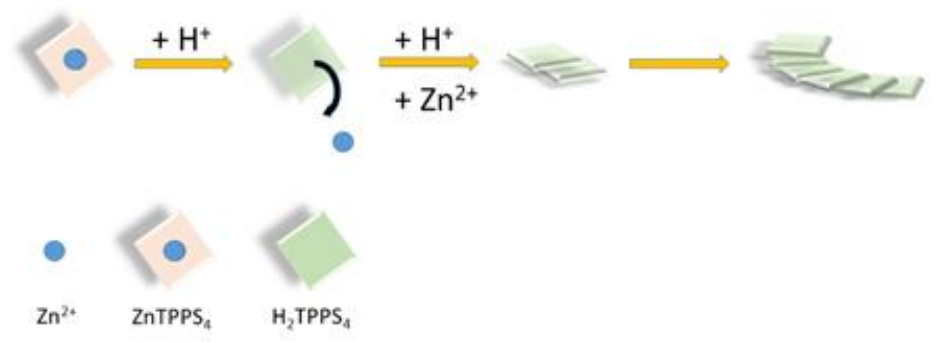

Scheme 1. Pictorial sketch of the basic strategy to growth J-aggregates of the diacid $\mathrm{H}_{2} \mathrm{TPPS}_{4}$ by demetallation of ZnTPPS $_{4}$ under acidic conditions.

This particular metal complex offers three main advantages: i) since the rate of zinc(II) extrusion from the porphyrin core is first order on $\left[\mathrm{ZnTPPS}_{4}\right]$ and second order on $\left[\mathrm{H}^{+}\right]$, the proper choice of pH and ionic strength conditions allows a fine time-control on the initial concentration of monomeric diacid $\mathrm{H}_{2}$ TPPS $_{4}$. Actually, by lowering the $\mathrm{pH}$ the demetallation becomes fast with respect to the J-aggregate formation, disentangling the two processes; ii) zinc(II) is usually a penta-coordinated metal ion and the presence of an axial ligand (usually water) on top of the porphyrin plane hinders formation of dimers or oligomers for this porphyrin; iii) the difference always observed in kinetics and optical activity when using different mixing protocols is removed. Purrello et al. exploited this strategy to induce chirality into a porphyrin supramolecular assembly pre-organized onto a polymeric support [26].

In this report, we have performed a detailed kinetic study of the demetallation of ZnTPPS and the subsequent aggregation into J-aggregates of the diacid $\mathrm{H}_{2} \mathrm{TPPS}_{4}$ generated in situ as function of $\mathrm{pH}$ and ionic strength. The main spectroscopic and chiroptical features of the J-aggregates have been related to the experimental conditions, suggesting an effective role of zinc cations.

\section{Kinetic analysis}

Our kinetic experiments have shown that when $\mathrm{ZnTPPS}_{4}$ is used as starting material, the rates are independent on the mixing order, and sigmoidal profiles for extinction vs time have been observed in all cases. A precise control on the release of metal free porphyrin could be achieved at pH higher than 1, a condition that requires rather high ionic strength to trigger aggregation. Since both the demetallation and aggregation rates could be influenced by the nature of the various species in solution, we used $\mathrm{H}_{2} \mathrm{SO}_{4}$ as acid to initiate both process and $\mathrm{ZnSO}_{4}$ to ensure the proper ionic strength. The spectral changes in the extinction spectra show the conversion of the starting ZnTPPS $_{4}$ into the intermediate diacid $\mathrm{H}_{2} \mathrm{TPPS}_{4}$ that eventually converts to J-aggregates. An analysis of the spectral changes allowed the calculation of the relevant kinetic parameters for both processes. The removal of the metal ion from its porphyrin complex follows the rate law: rate $=k_{2}\left[\mathrm{ZnTPPS}_{4}\right]\left[\mathrm{H}^{+}\right]^{2}$, with $k_{2}=5.5 \pm 0.4 \mathrm{M}^{-2} \mathrm{~s}^{-1}$ at $298 \mathrm{~K}$, in line with the literature ${ }^{[27]}$. The subsequent aggregation kinetics have been treated according to an established model proposed by Pasternack et al [28]. The rate determining step is the formation of a nucleus containing $m$ porphyrin units, that initiates the autocatalytic growth of the final assembly following a time-dependent process. The values of the catalytic rate constant $k_{c}$ monotonically increase on increasing both $\left[\mathrm{H}^{+}\right]$and ionic strength, while the value of $m$ indicates a trimer or a tetramer as the 
initial nucleus.

\section{Chiroptical properties}

Samples of the prepared J-aggregates exhibit rather peculiar spectroscopic features: i) a broad J-band, suggesting a dipolar coupling mechanism, instead of the usual Frenkel exciton model [299][이][피], ii) resonance enhanced light scattering (RLS) corresponding to the absorption bands due to their size and the strong electronic coupling among the monomers ${ }^{[32]}$. The increase of intensity of RLS on increasing $\left[\mathrm{H}^{+}\right]$and ionic strength suggests that aggregates become larger at lower $\mathrm{pH}$ and higher ionic strength. As already reported in literature, spontaneous symmetry breaking can occur in these systems, leading to the observation of circular dichroism (CD) spectra [20][21] . On increasing $\left[\mathrm{H}^{+}\right]$or the ionic strength, i.e. increasing the aggregation rates (see Figure 1), the absolute intensity of the dissymmetry g-factor decreases and the bands eventually switch from negative to positive Cotton effect.

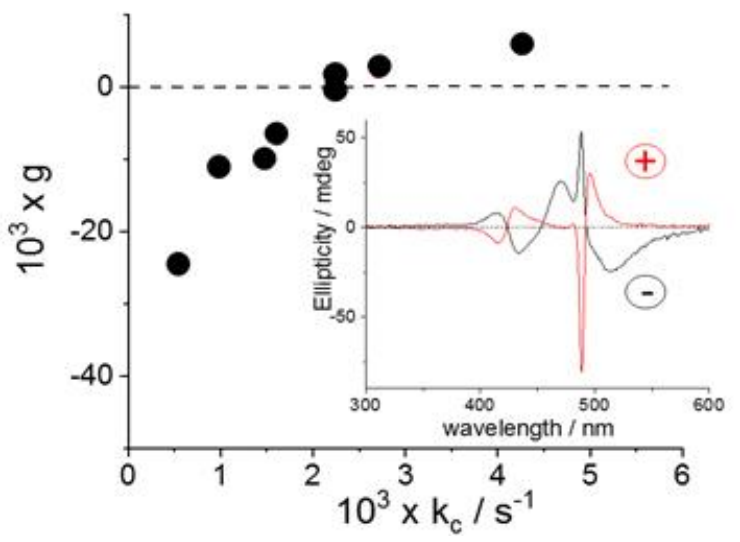

Figure 1. Plot of dissymmetry $g$-factor as function of the corresponding rate constants $k_{c}$ and two representative $\mathrm{CD}$ spectra for positive and negative Cotton effect (inset).

All our experimental evidence points to a specific role played by the zinc(II) ions, at molecular level and on the mesoscopic structure of the aggregates, in analogy to other polycationic species [29][30][31] At difference with J-aggregates prepared by simply adding inorganic acids, the sign of the Cotton effect in these species can be changed from negative to positive by varying $\mathrm{pH}$ and ionic strength [19][22]. All together, these findings shed further light onto these fascinating supramolecular assemblies and pave the way to new approaches in order to modulate their spectroscopic and chiroptical properties for potential applications.

\section{References}

1. Kobayashi, T. . J-aggregates; Kobayashi, T., Eds.; World Scientific Publishing Company: sINGAPORE, $1996 ;$ pp. 1.

2. Kobayashi, T. . J-aggregates; Kobayashi, T. , Eds.; World Scientific Publishing Company: Singapore, 2012 ; pp. Vol. 2.

3. Micali, N.; Villari, V.; Castriciano, M.A.; Romeo, A.; Scolaro, L.M.; From fractal to nanorod porphyrin J-aggregates. Concentration-induced tuning of the aggregate size. Journal of Physical Chemistry B 2006, 110, 8289-8295, 10.1021/jp060730e.

4. Maria Angela Castriciano; Andrea Romeo; Valentina Villari; Norberto Micali; Luigi Monsù Scolaro; Structural Rearrangements in 5,10,15,20-Tetrakis(4-sulfonatophenyl)porphyrin J-Aggregates under Strongly Acidic Conditions. The Journal of Physical Chemistry B 2003, 107, 8765-8771, 10.1021/jp0273880.

5. Norberto Micali; Francesco Mallamace; Andrea Romeo; Roberto Purrello; Luigi Monsù Scolaro; Mesoscopic Structure ofmeso-Tetrakis(4-sulfonatophenyl)porphine J-Aggregates. The Journal of Physical Chemistry B 2000, 104, 58975904, 10.1021/jp991909a.

6. A.S.R Koti; Jharna Taneja; N Periasamy; Control of coherence length and aggregate size in the J-aggregate of porphyrin. Chemical Physics Letters 2003, 375, 171-176, 10.1016/s0009-2614(03)00866-2.

7. Ricardas Rotomskis; Ramūnas Augulis; Valentinas Snitka; Ramūnas Valiokas; Bo Liedberg; Hierarchical Structure of TPPS4J-Aggregates on Substrate Revealed by Atomic Force Microscopy. The Journal of Physical Chemistry B 2004, 108, 2833-2838, 10.1021/jp036128v.

8. Maria Angela Castriciano; Andrea Romeo; Roberto Zagami; Norberto Micali; Luigi Monsu'scolaro; Kinetic effects of 
tartaric acid on the growth of chiral J-aggregates of tetrakis(4-sulfonatophenyl)porphyrin. Chemical Communications 2012, 48, 4872, 10.1039/c2cc00028h.

9. Maria Angela Castriciano; Andrea Romeo; Giovanna De Luca; Valentina Villari; Luigi Monsù Scolaro; Norberto Micali; Scaling the Chirality in Porphyrin J-Nanoaggregates. Journal of the American Chemical Society 2011, 133, 765-767, 10.1021/ja110028g.

10. Roberto Purrello; Luigi Monsu'scolaro; Emanuele Bellacchio; Sergio Gurrieri; Andrea Romeo; Chiral H- and J-Type Aggregates of meso-Tetrakis(4-sulfonatophenyl)porphine on alpha-Helical Polyglutamic Acid Induced by Cationic Porphyrins. Inorganic Chemistry 1998, 37, 3647.

11. Li Zhang; Minghua Liu; Supramolecular Chirality and Chiral Inversion of Tetraphenylsulfonato Porphyrin Assemblies on Optically Active Polylysine. The Journal of Physical Chemistry B 2009, 113, 14015-14020, 10.1021/jp902870f.

12. Lizhi Zhao; Xin Wang; Yan Li; Rujiang Ma; Yingli An; Linqi Shi; Chiral Micelles of Achiral TPPS and Diblock Copolymer Induced by Amino Acids. Macromolecules 2009, 42, 6253-6260, 10.1021/ma900925q.

13. Zoubir El-Hachemi; Teodor Silviu Balaban; J. Lourdes Campos; Sergio Cespedes; Joaquim Crusats; Carlos Escudero; Christina S. Kamma-Lorger; Joan Llorens; Marc Malfois; Geoffrey R. Mitchell; et al.Ana P. Tojeirajosep M. Ribó Effect of Hydrodynamic Forces onmeso-(4-Sulfonatophenyl)-Substituted Porphyrin J-Aggregate Nanoparticles: Elasticity, Plasticity and Breaking. Chemistry - A European Journal 2016, 22, 9740-9749, 10.1002/chem.201600874.

14. Alessandro D'Urso; Rosalba Randazzo; Maria Letizia Lo Faro; Roberto Purrello; Vortexes and Nanoscale Chirality. Angewandte Chemie International Edition 2009, 49, 108-112, 10.1002/anie.200903543.

15. Josep M. Ribó; Joaquim Crusats; Francesc Sagués; Josep Claret; Raimon Rubires; Chiral Sign Induction by Vortices During the Formation of Mesophases in Stirred Solutions. Science 2001, 292, 2063-2066, 10.1126/science.1060835.

16. Joaquim Crusats; Zoubir El-Hachemi; Josep M. Ribó; Hydrodynamic effects on chiral induction. Chemical Society Reviews 2010, 39, 569-577, 10.1039/b916369g.

17. Norberto Micali; H. Engelkamp; P. G. Van Rhee; P. C. M. Christianen; L. Monsù Scolaro; J. C. Maan; Selection of supramolecular chirality by application of rotational and magnetic forces. Nature Chemistry 2012, 4, 201-207, 10.1038/nchem.1264.

18. Jiashu Sun; Yike Li; Fusheng Yan; Chao Liu; Yutao Sang; Fei Tian; Qiang Feng; Pengfei Duan; Li Zhang; Xinghua Shi; et al.Baoquan DingMinghua Liu Control over the emerging chirality in supramolecular gels and solutions by chiral microvortices in milliseconds.. Nature Communications 2018, 9, 2599, 10.1038/s41467-018-05017-7.

19. I. G. Occhiuto; R. Zagami; Mariachiara Trapani; L. Bolzonello; A. Romeo; Maria Angela Castriciano; E. Collini; Luigi Monsù Scolaro; The role of counter-anions in the kinetics and chirality of porphyrin J-aggregates. Chemical Communications 2016, 52, 11520-11523, 10.1039/c6cc05768c.

20. Judith M Short; John A. Berriman; Christian Kuebel; Zoubir El-Hachemi; Jean-Valère Naubron; Teodor Silviu Balaban; Electron Cryo-Microscopy of TPPS4.2 HCl Tubes Reveals a Helical Organisation Explaining the Origin of their Chiralityt. ChemPhysChem 2013, 14, 3209-3214, 10.1002/cphc.201300606.

21. Zoubir El-Hachemi; Carlos Escudero; Francisco Acosta-Reyes; M. Teresa Casas; Virginia Altoe; Shaul Aloni; Gerard Oncins; Alessandro Sorrenti; Joaquim Crusats; J. Lourdes Campos; et al.Josep M. Ribó Structure vs. properties chirality, optics and shapes - in amphiphilic porphyrin J-aggregates. Journal of Materials Chemistry C 2013, 1, 3337, 10.1039/c3tc30299g.

22. Andrea Romeo; Maria Angela Castriciano; Ilaria Occhiuto; Roberto Zagami; Robert F. Pasternack; Luigi Monsu'scolaro; Kinetic Control of Chirality in Porphyrin J-Aggregates. Journal of the American Chemical Society 2013, 136, 40-43, 10.1021/ja410514k.

23. Francesco Mallamace; Luigi Monsu'scolaro; Andrea Romeo; Norberto Micali; Crossover in the Kinetic Growth Process of Porphyrin Aggregation. Physical Review Letters 1999, 82, 3480-3483, 10.1103/physrevlett.82.3480.

24. A. Romeo; Maria Angela Castriciano; R. Zagami; G. Pollicino; L. Monsù Scolaro; R. F. Pasternack; Effect of zinc cations on the kinetics of supramolecular assembly and the chirality of porphyrin J-aggregates. Chemical Science 2017, 8 , 961-967, 10.1039/c6sc02686a.

25. Peter Hambright; The coordination chemistry of metalloporphyrins. Coordination Chemistry Reviews 1971, 6, 247268, 10.1016/s0010-8545(00)80041-7.

26. Massimiliano Gaeta; Domenica Raciti; Rosalba Randazzo; Chiara Maria Antonietta Gangemi; Antonio Raudino; Alessandro D'Urso; Maria Elena Fragalà; Roberto Purrello; Antonino Raudino; Chirality Enhancement of Porphyrin Supramolecular Assembly Driven by a Template Preorganization Effect. Angewandte Chemie International Edition 2018, 57, 10656-10660, 10.1002/anie.201806192.

27. S.K. Cheung; F.L. Dixon; E.B. Fleischer; D.Y. Jeter; M. Krishnamurthy; Kinetic studies of the formation, acid-catalyzed solvolysis, and cupric ion displacement of a zinc porphyrin in aqueous solutions. Bioinorganic Chemistry 1973, 2 , 281-294, 10.1016/s0006-3061(00)80201-x.

28. Robert F. Pasternack; Cavan Fleming; Stephanie Herring; Peter J. Collings; Julio DePaula; Gerard DeCastro; Esther J. Gibbs; Aggregation kinetics of extended porphyrin and cyanine dye assemblies.. Biophysical Journal 2000, 79, 550- 
560, 10.1016/s0006-3495(00)76316-8.

29. Roberto Zagami; Maria Angela Castriciano; Andrea Romeo; Mariachiara Trapani; Rolando Pedicini; Luigi Monsu'scolaro; Tuning supramolecular chirality in nano and mesoscopic porphyrin J-aggregates. Dyes and Pigments 2017, 142, 255-261, 10.1016/j.dyepig.2017.03.047.

30. Luigi Monsu'scolaro; Andrea Romeo; Maria Angela Castriciano; Norberto Micali; Unusual optical properties of porphyrin fractal J-aggregates. Chemical Communications 2005, 24, 3018, 10.1039/b501083g.

31. Norberto Micali; Valentina Villari; Luigi Monsu'scolaro; Andrea Romeo; Maria Angela Castriciano; Light scattering enhancement in an aqueous solution of spermine-induced fractal J -aggregate composite. Physical Review E 2005, 72, 050401(R), 10.1103/physreve.72.050401.

32. R. Pasternack; P. Collings; Resonance light scattering: a new technique for studying chromophore aggregation. Science 1995, 269, 935-939, 10.1126/science.7638615.

\section{Keywords}

Porphyrins; J-Aggregates; Symmetry breaking; Demetallation kinetics; Aggregation kinetics; Chiral supramolecular assemblies

\section{DOI Information}

DOI: 10.32545/encyclopedia202006.0019.v10 | ه PDF [ 482kb, updated 10 July 2020 ] 